\title{
Advanced Channel Estimation for MIMO-OFDM in Realistic Channel Conditions
}

\author{
Jos Akhtman and Lajos Hanzo \\ School of ECS., Univ. of Southampton, SO17 1BJ, UK. \\ Tel: +44-23-80-593 125, Fax: +44-23-80-593 045 \\ Email: 1h@ecs.soton.ac.uk, http://www-mobile.ecs.soton.ac.uk
}

\begin{abstract}
An advanced decision-directed channel estimation scheme is proposed, which is suitable for employment in a wide range of multi-antenna multi-carrier systems as well as for communications over the entire range of practical channel conditions. In particular, we consider a MIMO-OFDM system operating in a mobile wireless multipath channel, which exhibits frequency-selective Rayleigh fading and is characterized by a time-variant power delay profile. Both the mean square error as well as the bit error rate performances achieved by the proposed system are documented. Specifically, we report a virtually errorfree performance of a rate $\frac{1}{2}$ turbo-coded 8x8-QPSK-OFDM system, exhibiting an effective throughput of $8 \cdot 2 \cdot \frac{1}{2}=8 \mathrm{bits} / \mathrm{sec} / \mathrm{Hz}$, while having a pilot overhead of only $10 \%$, at an SNR of $10 \mathrm{~dB}$ and an OFDM-symbol-normalized Doppler frequency of 0.003 , which corresponds to the mobile terminal speed of about 65 $\mathbf{k m} / \mathbf{h}$.
\end{abstract}

\section{INTRODUCTION}

Both the academic and the industrial research communities have achieved substantial advances in the field of practical multiple-input multiple-output (MIMO) transceiver architecture [1]. Nonetheless, approaching the channel capacity in realistic channel conditions remains a challenging problem. In particular, a robust and accurate channel estimation in MIMO systems constitutes a major issue, preventing us from achieving the high capacities predicted by the relevant theoretical analysis.

The family of well-documented decision directed channel estimation (DDCE) methods [1]-[3] provides a suitable solution for the problem of channel estimation in OFDM-based systems. The major benefit of the DDCE scheme is that in contrast to purely pilot assisted channel estimation methods [4] both the pilot symbols as well as all the information symbols are utilised for channel estimation [1]. The simple philosophy of this method is that in the absence of transmission errors we can benefit from the availability of $100 \%$ pilot information by using the detected subcarrier symbols as an a posteriori reference signal. The employment of this method allows us to reduce the number of pilot symbols required.

The majority of the aforementioned channel estimation methods rely on the a priori knowlege of the channel statistics

Acknowledgements: The work reported in this paper has formed part of the Wireless Enabling Techniques work area of the Core 4 Research Programme of the Virtual Centre of Excellence in Mobile and Personal Communications, Mobile VCE, www.mobilevce.com, whose funding support, including that of EPSRC, is gratefully acknowledged. Fully detailed technical reports on this research are available to Industrial Members of Mobile VCE. commonly characterized by the channel's Power Delay Profile (PDP) for the sake of estimating the instantenious Channel Impulse Response and the corresponding frequency-domain Channel Transfer Function (CTF). It is evident however that in realistic wireless mobile channels, where at least one of the communicating terminals is in motion, the channel's PDP will also become time-variant and thus may not be a priori known at the receiver. In this paper we propose a MIMODDCE method, which employs the so-called Projection Approximation Subspace Tracking (PAST) algorithm [5] for the sake of recursive tracking of the channel's PDP and subsequent estimation of the instantaneous CTF.

\section{System Model}

\section{A. Single Antenna OFDM System Model}

The discrete frequency-domain model of the OFDM system can be described as in [6]

$$
y[n, k]=H[n, k] x[n, k]+w[n, k],
$$

for $k=0, \ldots, K-1$ and all $n$, where $y[n, k], x[n, k]$ and $w[n, k]$ are the received symbol, the transmitted symbol and the Gaussian noise sample respectively, corresponding to the $k$ th subcarrier of the $n$th OFDM block. Furthermore, $H[n, k]$ represents the complex-valued Channel Transfer Function (CTF) coefficient associated with the $k$ th subcarrier and time instance $n$. In the case of an $M$-QAM modulated OFDM system, $x[n, k]$ corresponds to the $M$-QAM symbol accommodated by the $k$ th subcarrier.

As it was pointed out in [3], in OFDM systems using a sufficiently long cyclic prefix and adequate synchronisation, the subcarrier-related CTF can be expressed as

$$
H[n, k] \triangleq H(n T, k \Delta f)=C(k \Delta f) \sum_{l=1}^{L} \alpha_{l}[n] W_{K}^{k \tau_{l} / T_{s}}
$$

where $\boldsymbol{\alpha}[n]$ is the fractionally-spaced CIR and $C(f)$ is an aggregate transfer function of the transmitter-receiver pulseshaping filter pair. Note that in realistic channel conditions associated with non-sample-spaced time-variant path-delays $\tau_{l}(n)$ the fractionally-spaced CIR (FS-CIR) $\alpha_{l}[n] \triangleq \alpha_{l}(n T)$ will be constituted by a low number of $L$ non-zero statistically independent taps associated with distinctive propagation paths. The corresponding PDP $\lambda_{l}[n]$ constituted by the Mean Square 
(MS) values $\mathrm{E}\left\{\left|\alpha_{l}[n]\right|^{2}\right\}$ may be identified as the vector of the eigenvalues of the channel's covariance matrix

$$
\boldsymbol{C}_{H}=\mathrm{E}\left\{\boldsymbol{H}[n] \boldsymbol{H}^{\mathrm{H}}[n]\right\}=\boldsymbol{W}[n] \operatorname{diag}\left(\lambda_{l}[n]\right) \boldsymbol{W}^{\mathrm{H}}[n],
$$

where $\boldsymbol{W}$ is a $(K \times L)$-dimensional matrix having the eigenvectors of the channel covariance matrix $C_{H}[n]$ as its columns. The columns of the transformation matrix $\boldsymbol{W}$ span the so-called signal subspace of the random vector process associated with the subcarrier-related CTF $\boldsymbol{H}[n]$. Equation (2) can be expressed in a matrix form as

$$
\boldsymbol{H}[n]=\boldsymbol{W}[n] \boldsymbol{\alpha}[n] .
$$

\section{B. MIMO-OFDM System Model}

We consider a MIMO wireless communication system employing $m_{\mathrm{t}}$ transmit and $n_{\mathrm{r}}$ receive antennas, hence, the corresponding MIMO wireless communication channel is constituted by $\left(n_{\mathrm{r}} \times m_{\mathrm{t}}\right)$ propagation links. Furthermore, each of the corresponding $\left(n_{\mathrm{r}} \times m_{\mathrm{t}}\right)$ single-input single-output (SISO) propagation links comprises multiple statistically independent components, termed as paths. Thus, each of these SISO propagation links can be characterised as a multipath SISO channel discussed in Section II-A. Similarly to the SISO case, the multi-carrier structure of our SDM-OFDM transceiver allows us to characterise the broadband frequency-selective channel considered as an OFDM subcarrier-related vector of flat-fading Channel Transfer Function (CTF) coefficients. However, as opposed to the SISO case, for each OFDM symbol $n$ and subcarrier $k$ the MIMO channel is characterized by a $\left(n_{\mathrm{r}} \times m_{\mathrm{t}}\right)$-dimensional matrix $\mathbf{H}[n, k]$ of the CTF coefficients associated with the different propagation links, such that the element $H_{i j}[n, k]$ of the CTF matrix $\mathbf{H}[n, k]$ corresponds to the propagation link connecting the $j$ th transmit and $i$ th receive antennas.

The corresponding per subcarrier discrete frequency-domain model of the MIMO-OFDM system can be expressed as follows

$$
\mathbf{y}[n, k]=\mathbf{H}[n, k] \mathbf{x}[n, k]+\mathbf{w}[n, k],
$$

where we introduce the space-devision-related vectors $\mathbf{y}[n, k]$, $\mathbf{x}[n, k]$ and $\mathbf{w}[n, k]$, as well as a space-devision-related $\left(n_{\mathrm{r}} \times\right.$ $m_{\mathrm{t}}$ )-dimensional matrix of CTF coefficients $\mathbf{H}[n, k]$. Note that similarly to the SISO case, the multi-carrier structure of the MIMO-OFDM transceiver allows us to represent the broadband frequency-selective MIMO channel as a subcarrierrelated vector of flat-fading MIMO-CTF matrices $\mathbf{H}[n, k]$.

\section{Channel Estimation For MiMO-OFDM}

The main challenge associated with the estimation of the MIMO-CTF coefficients in the context of multi-antenna multicarrier systems rests in the fact that, as opposed to the SISO scenario outlined in Section II-A, the estimation of the MIMOCTFs constitutes a highly rank-deficient problem. More specifically, we consider the MIMO-OFDM system model associated with the $k$-th subcarrier of the $n$-th MIMO-OFDM symbol and characterized by Equation (5). Let us assume a relatively simple MIMO scenario of having $m_{\mathrm{t}}=n_{\mathrm{r}}=4$ transmit and

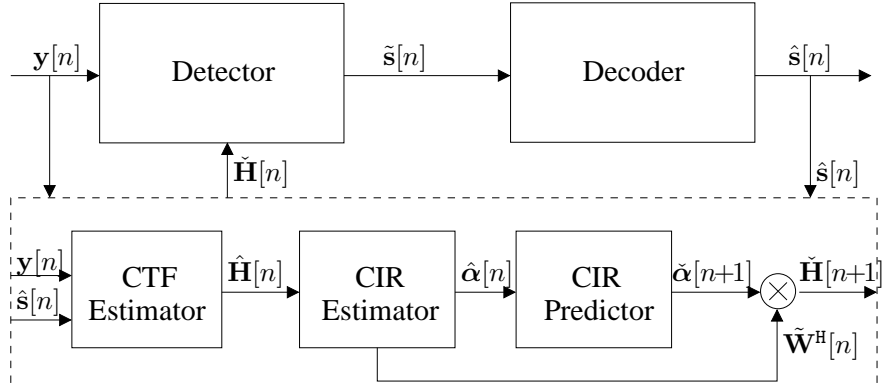

Decision Directed Channel Estimator

Fig. 1. Schematic of a generic receiver employing DDCE scheme constituted by an a posteriori decision-directed CTF Estimator, followed by a CIR Estimator and an a priori CIR predictor.

receive antennas. The corresponding MIMO-CTF matrix is constituted by $4 \times 4=16$ uncorrelated coefficients, which have to be calculated using four recorded samples comprising the received signal $\mathbf{y}[n, k]$, as well as four pilots or decision based symbols estimating the transmitted signal $\mathbf{s}[n, k]$. Notice that even in the presence of the a priori known pilot-based transmitted signal s $\mathbf{s}, k]$, the MIMO-CTF matrix $\boldsymbol{H}[n, k]$ may not be estimated reliably using a linear solution, such as for instance the MMSE method [7]. Consequently, the estimation of the $\left(n_{\mathrm{r}} \times m_{\mathrm{t}}\right)$-dimensional MIMO-CTF matrix $\mathbf{H}[n, k]$ requires a sufficiently sophisticated exploitation of both the time- and the frequency-domain correlation properties of the MIMO-CTF coefficients.

The schematic of the channel estimation method considered is depicted in Figure 1. Our channel estimator is constituted by an a posteriori decision-directed FD-CTF estimator followed by a parametric CIR etimator and an a priori CIR predictor [1]. As seen in Figure 1, the task of the CTF estimator is to calculate the temporary estimates $\tilde{\boldsymbol{H}}[n]$ of the CTF coefficients. Subsequently, the task of the CIR estimator is to track the a posteriori estimates $\hat{\alpha}_{l}[n]$ of the CIR taps of Equation (2), which are then fed into the low-rank timedomain CIR tap predictor of Figure 1 for the sake of producing an a priori estimate $\check{\alpha}_{l}[n+1], l=0,1, \cdots, L$ of the next CIR on a CIR tap-by-tap basis [1]. Finally, the predicted CIR is converted to the CTF with the aid of the tranformation matrix $\boldsymbol{W}[n]$ of Figure 1 . The resultant CTF is employed by the receiver for the sake of detecting and decoding of the next OFDM symbol.

\section{A. Soft Recursive MIMO-CTF Estimation}

At the first stage of our MIMO channel estimation scheme we employ an array of $K$ per-subcarrier MIMO-CTF estimators, which function independently of each other. Consequently, for the sake of notational simplicity we omit the subcarrier related index $k$ in the following section. The Recursive Least Squares (RLS) algorithm [7]-[9] constitutes a rapidly-converging least squares algorithm. The RLS attempts to minimize the cost function created from the exponentiallyweighted and windowed sum of the squared error. Namely, we 
have

$$
J_{\mathrm{RLS}}[n]=\sum_{m=1}^{n} \zeta^{n-m} \mathbf{e}^{\mathrm{H}}[m, n] \mathbf{e}[m, n],
$$

where, the error signal $\mathbf{e}[m, n]$ may be expressed as

$$
\mathbf{e}[m, n]=\mathbf{y}[m]-\tilde{\mathbf{H}}[n] \mathbf{s}[m],
$$

while $\zeta$ denotes the forgetting factor. The corresponding RLS estimate of the $\left(n_{\mathrm{r}} \times m_{\mathrm{t}}\right)$-dimensional MIMO-CTF coefficient matrix associated with the $k$ th subcarrier of the $n$th OFDM symbol may be calculated as follows [9]

$$
\tilde{\mathbf{H}}[n]=\left(\boldsymbol{\Phi}^{-1}[n] \boldsymbol{\theta}[n]\right)^{\mathrm{H}},
$$

where we define the MIMO-CTF estimator's input autocorrelation function $\boldsymbol{\Phi}[n]$, which may be calculated recursively as follows

$$
\mathbf{\Phi}[n]=\sum_{m=1}^{n} \zeta^{n-m} \mathbf{s}[m] \mathbf{s}^{\mathrm{H}}[m]=\zeta \boldsymbol{\Phi}[n-1]+\mathbf{s}[n] \mathbf{s}^{\mathrm{H}}[n],
$$

while the MIMO-CTF estimator's input-output crosscorrelation matrices $\boldsymbol{\theta}[n]$ may be expressed as

$$
\boldsymbol{\theta}[n]=\sum_{m=1}^{n} \zeta^{n-m} \mathbf{s}[m] \mathbf{y}^{\mathrm{H}}[m]=\zeta \boldsymbol{\theta}[n-1]+\mathbf{s}[n] \mathbf{y}^{\mathrm{H}}[n] .
$$

As suggested by the decision-directed philosophy of the channel estimation scheme outlined in Section III, the transmitted signal vector $\mathbf{s}[n]$ may not always be readily available at the receiver. More specifically, the transmitted signal vector $\mathbf{s}[n]$ may be assumed to be known a priori if and only if s constitutes a pilot symbol, which occupies a small portion of the transmitted data-stream. Alternatively, whenever an information-carrying data-symbol is transmitted, the decisionbased estimates $\hat{\boldsymbol{s}}[n]$ become available instead. Unfortunately, however, the decision-based estimates $\hat{\boldsymbol{s}}$ are prone to decision errors, which may potentially result in error propagation and thus in a substantial performance degradation.

Consequently, as pointed out in [8], [9], it is highly beneficial to exploit the probability-related soft information available at the output of the MIMO-OFDM system's detector. More specifically, in addition to the hard-decision based values of the transmitted signal estimates $\hat{\mathbf{s}}[n]$ we may utilize the associated soft-information-related quantities, such as the expectations of the elements of the estimated transmitted signal vector $\hat{\mathbf{s}}=\left[\hat{s}_{1}, \cdots, \hat{s}_{m_{\mathrm{t}}}\right]^{\mathrm{T}}$. Specifically, the expectation of the $i$ th transmitted symbol may be expressed as follows

$$
\tilde{s}_{i}=\mathrm{E}\left\{s_{i}\right\}=\sum_{c \in \mathcal{M}} c p\left\{s_{i}=c\right\} .
$$

Subsequently, we may define the following alternative error signals

$$
\begin{aligned}
\hat{\mathbf{e}}[m, n] & =\mathbf{y}[m]-\tilde{\mathbf{H}}[n] \hat{\mathbf{s}}[m], \\
\tilde{\mathbf{e}}[m, n] & =\mathbf{y}[m]-\tilde{\mathbf{H}}[n] \tilde{\mathbf{s}}[m] .
\end{aligned}
$$

The error signals of Equations (12) and (13) may be substituted into the RLS algorithms of Section III-A in order to yield the hard and soft decision-based RLS CTF tracking algorithms, respectively.

\section{B. PASTD Aided CIR Estimation}

Let $\boldsymbol{H}[n] \in \mathbb{C}^{K}$ be the vector of the subcarrier-related CTF coefficients associated with the channel model of Equation (1). As described in Section II, the CIR associated with the CTF coefficient vector $\boldsymbol{H}[n]$ is constituted by a relatively low number of $L \ll K$ statistically-independent Rayleigh fading paths. The corresponding CIR components are related to the CTF coefficients $H[n, k]$ by means of Equation (2). The motivation for employing the so-called subspace technique [10] here is that usually we have $L \ll K$ and thus it is more efficient to estimate a low number of CIR-related taps in the low-dimensional signal subspace than estimating all the $K$ FD-CTF coefficients.

An iterative RLS algorithm for tracking of the signal subspace of the channel's covariance matrix $\boldsymbol{C}_{H}$ of Equation (3) was proposed by Yang [5]. Specifically, let us consider the following RLS objective function

$$
J(\boldsymbol{W}[n])=\sum_{m=1}^{n} \eta^{n-m}\left\|\boldsymbol{H}[m]-\boldsymbol{W}[n] \boldsymbol{W}^{\mathrm{H}}[n] \boldsymbol{H}[m]\right\|^{2},
$$

where $\eta \in(0,1)$ is the so-called forgetting factor, which accounts for possible deviations of the actual channel statistics encountered from the WSS assumption.

The PAST algorithm [5] may be derived by approximating the expression $\boldsymbol{W}^{\mathrm{H}}[n] \boldsymbol{H}[m]$ in Equation (14), which may be interpreted as a projection of the vector $\boldsymbol{H}[\mathrm{m}]$ onto the column space of the matrix $\boldsymbol{W}[n]$, by the readily available $a$ posteriori vector $\boldsymbol{\alpha}[m]=\boldsymbol{W}^{\mathrm{H}}[m] \boldsymbol{H}[m]$. The resultant modified cost function may be formulated as

$$
J^{\prime}(\boldsymbol{W}[n])=\sum_{m=1}^{n} \eta^{n-m}\|\boldsymbol{H}[m]-\boldsymbol{W}[n] \boldsymbol{\alpha}[m]\|^{2} .
$$

As is argued in [5], for stationary or slowly varying signals, the aforementioned projection approximation, hence the name PAST, does not substantially change the error surface associated with the corresponding cost function of Equation (15) and therefore does not significantly affect the convergence properties of the derived algorithm.

Following the RLS approach [5], a low-complexity solution of the computational problem associated with minimizing the cost function $J^{\prime}(\boldsymbol{W}[n])$ of Equation (15) may be obtained using recursive updates of the matrix $\boldsymbol{W}[n]$. More specifically, we have

$$
\boldsymbol{W}[n]=\boldsymbol{W}[n-1]+\boldsymbol{e}[n] \boldsymbol{k}^{\mathrm{H}}[n],
$$

where $e[n]$ is the estimation error vector, which may be recursively obtained as

$$
\boldsymbol{e}[n]=\boldsymbol{H}[n]-\boldsymbol{W}[n-1] \boldsymbol{\alpha}[n-1],
$$

while

$$
\boldsymbol{k}[n]=\frac{\boldsymbol{P}[n-1] \boldsymbol{\alpha}[n]}{\eta+\boldsymbol{\alpha}^{\mathrm{H}}[n] \boldsymbol{P}[n-1] \boldsymbol{\alpha}[n]}
$$

denotes the RLS gain vector. Furthermore, the matrix $\boldsymbol{P}[n]$ is the inverse of the CIR-related taps' $(L \times L)$-dimensional 
covariance matrix $C_{\alpha \alpha}$, which can be recursively calculated as follows

$$
\boldsymbol{P}[n]=\frac{1}{\eta} \operatorname{Tri}\left\{\left(\boldsymbol{I}-\boldsymbol{k}[n] \boldsymbol{\alpha}^{\mathrm{H}}[n]\right) \boldsymbol{P}[n-1]\right\},
$$

where the operator $\operatorname{Tr} i\{\cdot\}$ indicates that only the upper triangular part of $P[n]$ is calculated and its Hermitian conjugate version is copied to the lower triangular part [5].

In this paper, however, we aim for maintaining the lowest possible complexity hence we are particularly interested in the deflation-based version of the PAST algorithm derived in [5], which is referred to as the PASTD algorithm. The simple philosophy of the deflation method is the sequential estimation of the principal components of the CTF covariance matrix $\boldsymbol{C}_{H}$ [11]. Consequently, we first update the most dominant eigenvector $\boldsymbol{w}_{1}[n]$ by applying the PAST method in conjunction with $L=1$. Subsequently, the projection of the current sample vector $\boldsymbol{H}[n]$ onto the updated eigenvector $\boldsymbol{w}_{1}[n]$ is subtracted from itself, resulting in a modified (deflated) version of the CTF vector in the following form $\boldsymbol{H}_{2}[n]=$ $\boldsymbol{H}[n]-\boldsymbol{w}_{1}[n] \boldsymbol{w}_{1}^{\mathrm{H}}[n] \boldsymbol{H}[n]$. The second most dominant eigenvector $\boldsymbol{w}_{2}[n]$ has now become the most dominant one and therefore may be updated similarly to $\boldsymbol{w}_{1}[n]$. By repeatedly applying this procedure, all the desired eigencomponents may be estimated.

A particularly important property of the PASTD method is that as opposed to the PAST method, it enables the explicit tracking of the time-variant eigencomponents of the channel covariance matrix $\boldsymbol{C}_{H}[n]$, namely the eigenvectors $\boldsymbol{w}_{l}[n]$ as well as of the corresponding eigenvalues $\lambda_{l}[n]$ according to

$$
\boldsymbol{w}_{l}[n]=\boldsymbol{w}_{l}[n-1]+\frac{\alpha_{l}^{*}[n]}{\lambda_{l}[n]}\left(\boldsymbol{H}_{l}[n]-\boldsymbol{w}_{l}[n-1] \alpha_{l}[n]\right),
$$

where we have $\boldsymbol{\alpha}_{l}[n]=\boldsymbol{w}_{l}^{\mathrm{H}}[n-1] \boldsymbol{H}[n]$ and $\lambda_{l}[n]=\beta \lambda_{l}[n-$ $1]+\left|\alpha_{l}[n]\right|^{2}$.

\section{RLS CIR Tap Prediction}

Following the RLS-based adaptive CIR tap prediction approach of [12], [13], the time-variant $l$ th CIR tap's predictor filter coefficient vector $\boldsymbol{q}_{l}[n]$ is calculated by minimizing the following scalar cost function

$$
J\left(\alpha_{l}\right)=\sum_{i=1}^{n} \beta^{n-i}\left|\alpha_{l}[i+1]-\boldsymbol{q}_{l}^{\mathrm{H}}[n] \boldsymbol{\alpha}_{l}[i]\right|^{2},
$$

where $\beta \in(0,1)$ is the so-called forgetting factor [12], which accounts for possible deviations of the fading process encountered from the WSS assumption. The resultant recursive update for $\boldsymbol{q}_{l}[n]$ is given by

$$
\boldsymbol{q}_{l}[n]=\boldsymbol{q}_{l}[n-1]+\boldsymbol{k}_{l}[n-1] e_{l}^{*}[n],
$$

where

$$
e_{l}[n]=\hat{\alpha}_{l}[n]-\boldsymbol{q}_{l}^{\mathrm{H}}[n-1] \hat{\boldsymbol{\alpha}}_{l}[n-1]
$$

is the prediction error, while

$$
\boldsymbol{k}_{l}[n]=\frac{\boldsymbol{P}_{l}[n-1] \hat{\boldsymbol{\alpha}}_{l}[n]}{\beta+\hat{\boldsymbol{\alpha}}_{l}^{\mathrm{H}}[n] \boldsymbol{P}_{l}[n-1]_{l} \hat{\boldsymbol{\alpha}}_{l}[n]}
$$

denotes the RLS gain vector. Furthermore, the matrix $\boldsymbol{P}_{l}[n]$ is the inverse of the $l$ th CIR tap's $\left(N_{\text {prd }} \times N_{\text {prd }}\right)$-dimensional sample covariance matrix, which can be recursively calculated as follows

$$
\boldsymbol{P}_{l}[n]=\frac{1}{\beta}\left(\boldsymbol{I}-\boldsymbol{k}_{l}[n] \hat{\boldsymbol{\alpha}}_{l}^{\mathrm{H}}[n]\right) \boldsymbol{P}_{l}[n-1]
$$

As it was pointed out in [13] the choice of the forgetting factor's value $\beta$ has only a moderate effect on the performance of the resultant predictor. Specifically, in our simulations we used the value suggested in [13], namely $\beta=0.9$.

\section{PASTD -Aided DDCE for MIMO-OFDM}

The detailed structure of our MIMO-DDCE channel estimator, which follows the decision-directed channel estimation philosophy of Figure 1, is illustrated in Figure 2. Specifically, our MIMO channel estimation scheme comprises an array of $K$ per-subcarrier MIMO-CTF estimators of Section IIIA, followed by a $\left(n_{\mathrm{r}} \times m_{\mathrm{t}}\right)$-dimensional array of parametric CIR estimators of Section III-B and a corresponding array of $\left(n_{\mathrm{r}} \times m_{\mathrm{t}} \times L\right)$ CIR tap predictors of Section III-C, where $L$ is the number of tracked CIR taps per link for the MIMO channel.

\section{Performance Analysis and Conclusions}

TABLE I

SYSTEM PARAMETERS

\begin{tabular}{|l|r|}
\hline Parameter & Value \\
\hline \hline Carrier frequency $f_{c}$ & $2.5 \mathrm{GHz}$ \\
\hline Channel bandwidth $B$ & $8 \mathrm{MHz}$ \\
\hline Number of carriers $K$ & 128 \\
\hline FFT frame duration $T_{s}$ & $16 \mu \mathrm{s}$ \\
\hline OFDM symbol duration $T$ & $20 \mu \mathrm{s}(4 \mu \mathrm{s}$ of cyclic prefix $)$ \\
\hline Max. delay spread $\tau_{\max }$ & $4 \mu \mathrm{s}$ \\
\hline Max. terminal speed $v$ & $130 \mathrm{~km} / \mathrm{h}$ \\
\hline Norm. Max. Doppler spread $f_{D}$ & $0.006=T \cdot 300 \mathrm{~Hz}$ \\
\hline
\end{tabular}

In this section we would like to characterize the achievable performance of the MIMO-DDCE scheme of Figure 2in the context of the MIMO-OFDM system. More specifically, we consider a 2x2 MIMO-QPSK-OFDM system having $K=$ 128 orthogonal QPSK-modulated subcarriers. The system parameters are outlined in Table I. We employ an OFDMframe-variant channel model having the time-variant 7-tap PDP characterized by the COST-207 BU channel model of [14]. Additionally, each individual propagation path undergoes fast Rayleigh fading at an OFDM-symbol-normalized Doppler frequencie of $f_{D}=0.005$.

Firstly, Figure 3 characterizes the achievable MSE performance of the MIMO-DDCE method of Figure 2 for different values of the MIMO-CTF tracking scheme's forgetting factor $\zeta$. As may be concluded from Figure 3, the optimum value of the forgetting factor $\zeta$ is largely dependent on the SNR as well as on the Doppler frequency encountered. Nevertheless, the compromise value of $\zeta=0.7$ appears to constitute a relatively good choice in the practical range of SNR values and Doppler frequencies.

Secondly, Figure 4 characterizes the achievable MSE performance of the MIMO-DDCE method of Figure 2for different 


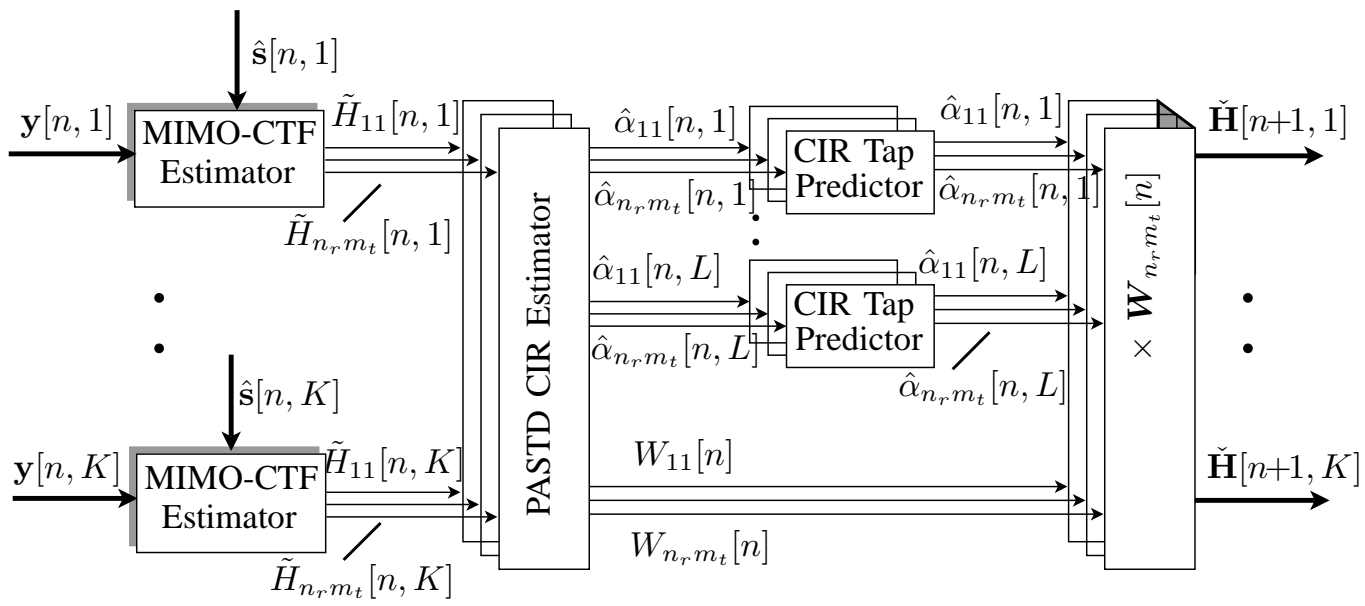

Fig. 2. Detailed structure of the MIMO channel estimator corresponding to the DDCE module of Figure 1 in the context of the MIMO-OFDM system. The channel estimator comprises an array of PASTD modules, which performs recursive tracking of the MIMO-CIR. The resultant MIMO-CIR related taps $\hat{\alpha}_{i j ; l}[n]$ are filtered by an array of adaptive RLS prediction filters resulting in the a priori estimates of the MIMO-CIR-related taps $\check{\alpha}_{i j ; l}[n+1]$. Finally, the a priori estimates of the subcarrier-related coefficients $\breve{\mathbf{H}}[n+1, k]$ are obtained by applying the array of transform matrices $\boldsymbol{W}_{i j}[n]$ provided by the PASTD modules.

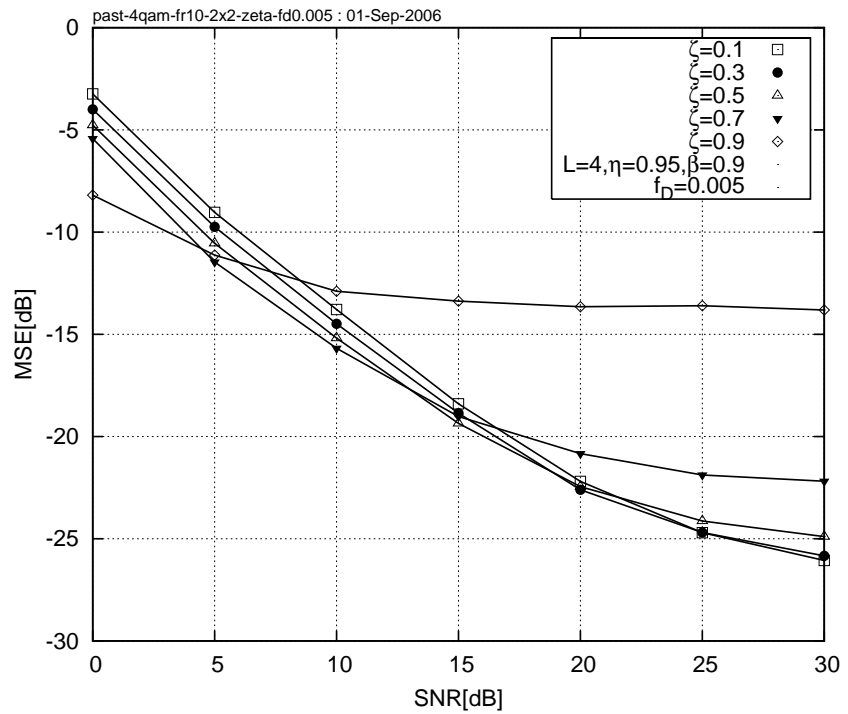

Fig. 3. MSE versus SNR performance exhibited by the 2x2 SDM-4QAMOFDM system employing the PASTD-aided MIMO-DDCE scheme of Figure 2. The PASTD-DDCE parameters were $\zeta=0.1,0.3, \cdots, 0.9$ as well as $\eta=0.95, \beta=0.9$. The Doppler frequency was $f_{D}=0.005$. Additional system parameters are summarized in Table I.

values of the PASTD aided CIR tracking scheme's forgetting factor $\eta$. Similarly to the choice of the optimum MIMOCTF tracking forgetting factor $\zeta$, the optimum value of the PASTD aided CIR tracking forgetting factor $\eta$ is largely dependent on the SNR as well as on the Doppler frequency encountered and the compromise value of $\eta=0.95$ appears to constitute a good choice across the practical range of SNR values and Doppler frequencies.

Furthermore, Figure 5 characterizes the achievable MSE performance of the MIMO-DDCE method of Figure 2for different ranks $L$ of the PASTD aided CIR tracking-related estimated subspace, while assuming a constant value of the

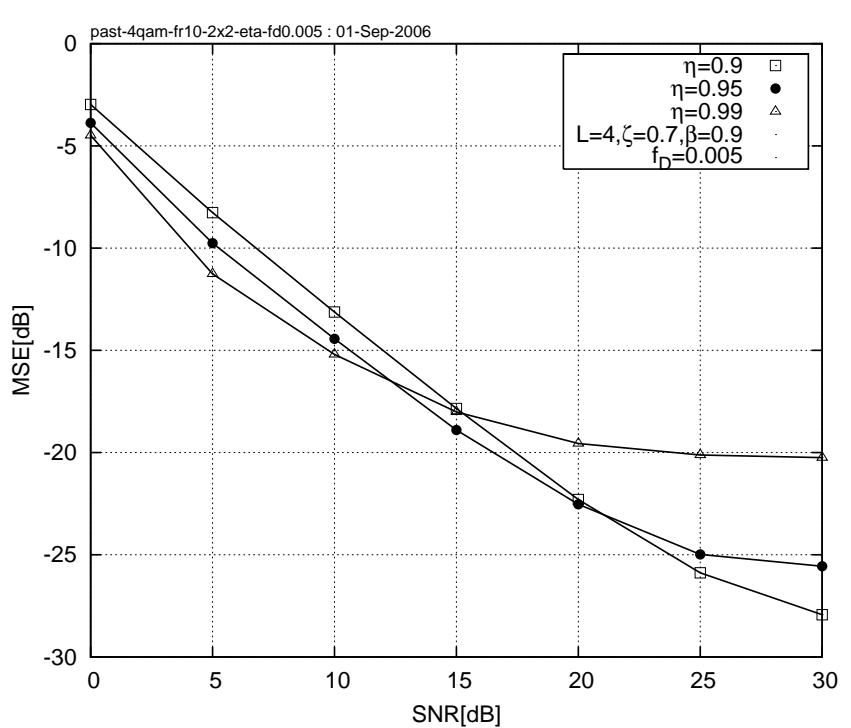

Fig. 4. MSE versus SNR performance exhibited by the $2 \times 2$ SDM-4QAMOFDM system employing the PASTD-aided MIMO-DDCE scheme of Figure 2. The PASTD-DDCE parameters were $\eta=0.9,0.95$ and 0.99 as well as $\zeta=0.7, \beta=0.9$. The Doppler frequency was $f_{D}=0.005$. Additional system parameters are summarized in Table I.

forgetting factors $\eta=0.95$ and $\zeta=0.7$. From Figure 5 we may conclude that a relatively high performance of the PASTD aided CIR estimator may be achieved when assuming that the rank of the estimated CTF signal subspace is $L=4$, regardless of the actual number of paths constituting the multipath CIR encountered.

Finally, Figure 6 characterizes the achievable BER performance of the rate $\frac{1}{2}$ turbo-coded SDM-QPSK-OFDM system employing the MIMO-PASTD-DDCE method of Figure 2. The DDCE parameters are $\zeta=0.7, L=4, \eta=0.95$ and $\beta=0.9$. Furthermore, we assumed a pilot overhead of $10 \%$. Figures $6(\mathrm{a})$ and 6 (b) correspond to the $4 \times 4$ and $8 \times 8$ 


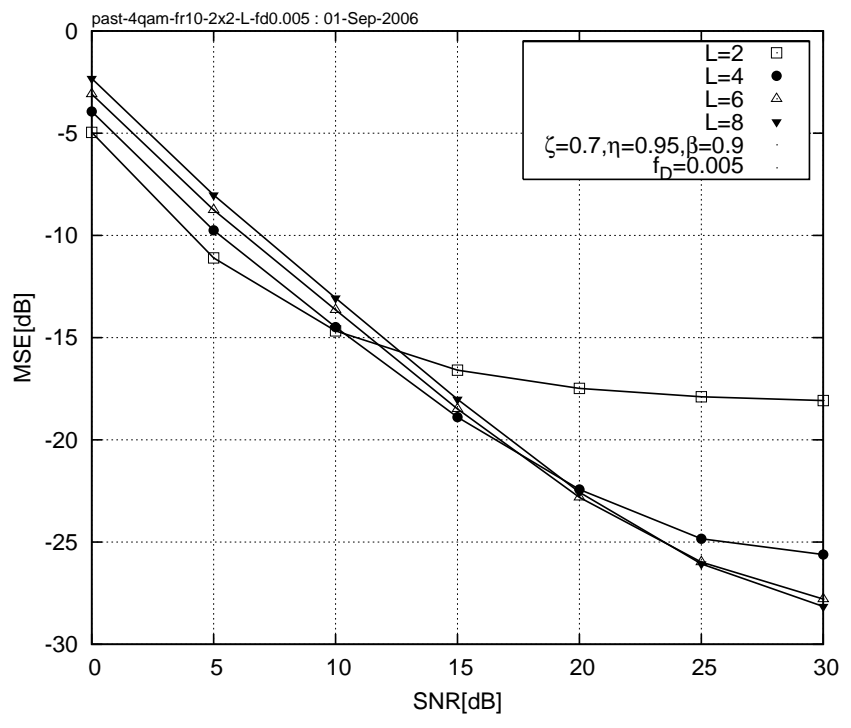

Fig. 5. MSE versus SNR performance exhibited by the $2 \times 2$ SDM-4QAMOFDM system employing the PASTD-aided MIMO-DDCE scheme of Figure 2and tracking $L=2,4,6$ and 8 CIR taps. The PASTD-DDCE parameters are $\zeta=0.7, \eta=0.95$ and $\beta=0.9$. The Doppler frequency was $f_{D}=0.005$. Additional system parameters are summarized in Table I.

MIMO scenarios, respectively. We considered encountering the OFDM-symbol-normalized Doppler frequencies of $f_{D}=$ $0.001,0.003$ and 0.005 . Observe, that the system proposed attains a virtually error-free performance of a rate $1 / 2$ turbocoded $8 \times 8$-QPSK-OFDM system, exhibiting a total bit rate of $8 \mathrm{bits} / \mathrm{s} / \mathrm{Hz} \cdot 8 \mathrm{MHz}=64 \mathrm{Mbps}$ and having a pilot overhead of only $10 \%$, at SNR of $10 \mathrm{~dB}$ and normalized Doppler frequency of 0.003 , which corresponds to the mobile terminal speed of roughly $65 \mathrm{~km} / \mathrm{h}^{1}$.

Our future research is related to reducing the pilot-overhead required, potentially leading to semi-blind channel estimation schemes.

\section{REFERENCES}

[1] L. Hanzo, M. Münster, B. J. Choi, and T. Keller, $O F D M$ and MC-CDMA for Broadband Multi-User Communications, WLANs and Broadcasting. John Wiley and IEEE Press, 2003, 992 pages.

[2] O. Edfords, M. Sandell, J.-J. van de Beek, S. Wilson, and P. Börjesson, "OFDM channel estimation by singular value decomposition," IEEE Transactions on Communications, vol. 46, pp. 931-939, July 1998.

[3] Y. Li, L. Cimini, and N. Sollenberger, "Robust channel estimation for OFDM systems with rapid dispersive fading channels," IEEE Transactions on Communications, vol. 46, no. 7, pp. 902-915, April 1998.

[4] Y. Li, "Pilot-symbol-aided channel estimation for OFDM in wireless systems," IEEE Transactions on Vehicular Technology, vol. 49, pp. $1207-1215$, July 2000.

[5] B. Yang, "Projection approximation subspace tracking," IEEE Transactions on Signal Processing, vol. 43, no. 1, pp. 95-107, January 1995.

[6] Y. Li, "Simplified channel estimation for OFDM systems with multiple transmit antennas," IEEE Transactions on Wireless Communications, vol. 1, no. 1, pp. 67-75, January 2002.

[7] S. M. Kay, Fundamentals of Statistical Signal Processing. Englewood Cliffs, NJ, USA: Prentice-Hall, 1998.

[8] M. Sandell, C. Luschi, P. Strauch, and R. Yan, "Iterative channel estimation using soft decision feedback," in Global Telecommunications Conference, 1998. GLOBECOM 98. The Bridge to Global Integration. IEEE, vol. 6, Sydney,NSW, 1998, pp. 3728-3733.

${ }^{1}$ Additional system parameters are characterized in Table I.

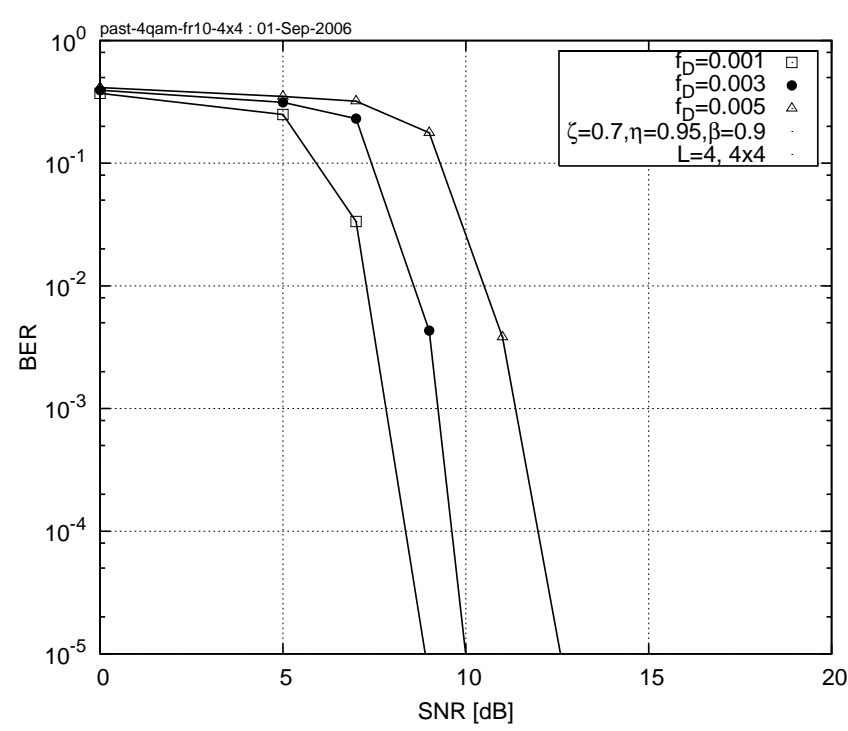

(a)

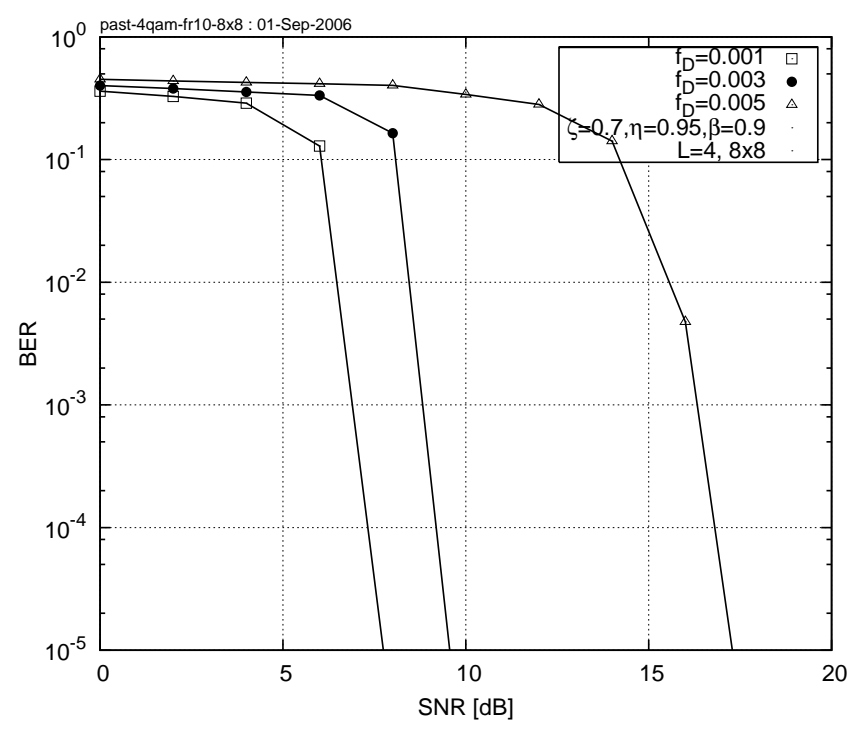

(b)

Fig. 6. BER versus SNR performance exhibited by the rate $\frac{1}{2}$ turbo-coded (a) $4 \times 4$ and (b) 8x8 SDM-QPSK-OFDM system employing the MIMO-PASTDDDCE method of Figure 2. Additional system parameters are summarized in Table I.

[9] R. Otnes and M. Tüchler, "Iterative channel estimation for turbo equalization of time-varying frequency-selective channels," IEEE Transactions on Wireless Communications, vol. 3, no. 6, pp. 1918-1923, 2004

[10] B. Yang, K. Letaief, R. Cheng, and Z. Cao, "Channel estimation for OFDM transmission in multipath fading channels based on parametric channel modeling," IEEE Transactions on Communications, vol. 49, pp. 467-479, March 2001.

[11] J. Yang and M. Kaveh, "Adaptive eigensubspace algorithm for direction or frequency estimation and tracking," IEEE Transactions on Acoustics, Speech and Signal Processing, vol. 36, pp. 241-251, 1988.

[12] S. Haykin, Adaptive Filter Theory. Englewood Cliffs, NJ, USA Prentice-Hall, 1996.

[13] D. Schafhuber and G. Matz, "MMSE and adaptive prediction of timevarying channels for OFDM systems," IEEE Transactions on Wireless Communications, vol. 4, no. 2, pp. 593-602, March 2005.

[14] M. Failli, "Digital land mobile radio communications COST 207," European Commission, Tech. Rep., 1989. 